\title{
Reemplazo total de cadera en un paciente con amputación supracondílea homolateral
}

\author{
Edgar G. Wagner, Reynaldo Quiroga \\ Servicio de Ortopedia y Traumatología, Hospital Regional de Comodoro Rivadavia, Chubut, Argentina
}

\section{RESUMEN}

Las fracturas de cadera en pacientes con amputación del miembro homolateral son lesiones infrecuentes que representan un reto para el cirujano ortopédico. Presentamos el caso de un hombre de 63 años con amputación supracondílea izquierda, que sufre una fractura de cuello femoral izquierdo por caída de su propia altura. La colocación apropiada del implante es esencial para obtener buenos resultados clínicos posoperatorios. Para la exposición y la colocación del implante se utilizó un clavo de Steinmann en el trocánter mayor. No se observaron complicaciones clínicas ni radiográficas en un seguimiento de 15 meses. Creemos que el empleo del clavo de Steinmann colocado en una correcta posición es una buena alternativa por considerar para poder controlar el miembro al realizar una artroplastia y así disminuir los riesgos de colocar la prótesis en mala posición y el tiempo quirúrgico. Palabras clave: Artroplastia de cadera; amputación supracondílea homolateral; reemplazo total de cadera. Nivel de Evidencia: IV

\section{Total hip arthroplasty in a patient with ipsilateral transfemoral amputation}

\begin{abstract}
Hip fractures in patients with ipsilateral limb amputations are uncommon injuries that pose a challenge for orthopedic surgeons. In this article, we present our experience in the treatment of a 63-year man with left transfemoral amputation, who suffered a left femoral neck fracture after a fall from standing height. Proper placement of the prosthesis is essential to achieve good postoperative clinical outcomes. A Steinmann pin was inserted into the greater trochanter to facilitate exposure and component placement. No clinical or radiological complications were detected in a 15-month follow-up. We believe that the correct placement of a Steinmann pin is a good alternative that provides adequate control over the extremity when performing an arthroplasty, thus reducing the risks for prosthesis displacement and shortening the surgical time.
\end{abstract}

Key words: Hip replacement; ipsilateral transfemoral amputation; total hip arthroplasty.

Level of Evidence: IV

\section{INTRODUCCIÓN}

Las fracturas de cadera son una fuente común de morbilidad y mortalidad en el mundo. Sin embargo, el descubrimiento y desarrollo de la artroplastia de cadera mejoraron el pronóstico de los pacientes, con una alta tasa de supervivencia y resultados funcionales satisfactorios. ${ }^{1-7}$

El reemplazo de cadera en pacientes sanos está muy bien descrito en la literatura, pero hemos encontrado poco material respecto al reemplazo en pacientes con amputación del miembro homolateral..$^{1-7}$

Respecto a los pacientes con amputación supracondílea, se ha observado un aumento del índice de fractura de cadera homolateral como consecuencia de la disminución de la densidad mineral ósea, principalmente en aquellos que tienen una ortesis. ${ }^{6}$

La fractura de cadera en pacientes con amputación de miembro homolateral es una lesión infrecuente que plantea un reto para el cirujano ortopédico debido a las características del paciente, las dificultades técnicas para su tratamiento, el abordaje quirúrgico y el manejo del muñón.,4,7

Recibido el 16-2-2019. Aceptado luego de la evaluación el 8-4-2019 • Dr. EDGAR G. WAGNER • edgar.gw@ hotmail.com

Cómo citar este artículo: Wagner EG, Quiroga R. Reemplazo total de cadera en un paciente con amputación supracondílea homolateral. Rev Asoc Argent Ortop Traumatol 2020;85(2):151156. https://doi.org/10.15417/issn.1852-7434.2020.85.2.964 
Comunicamos un caso raro de fractura de cuello femoral en un paciente con amputación supracondílea del mismo miembro, de un año de evolución, tratado, de forma primaria, mediante artroplastia total de cadera.

\section{CASO CLÍNICO}

Hombre de 63 años, diabético e hipertenso en tratamiento, con amputación supracondílea de miembro inferior izquierdo, de un año de evolución, que concurrió a la Guardia del Hospital tras sufrir un traumatismo de cadera izquierda por caída de su propia altura. Se le diagnosticó fractura de cuello femoral grado 2 según la clasificación de Garden (Figura 1).

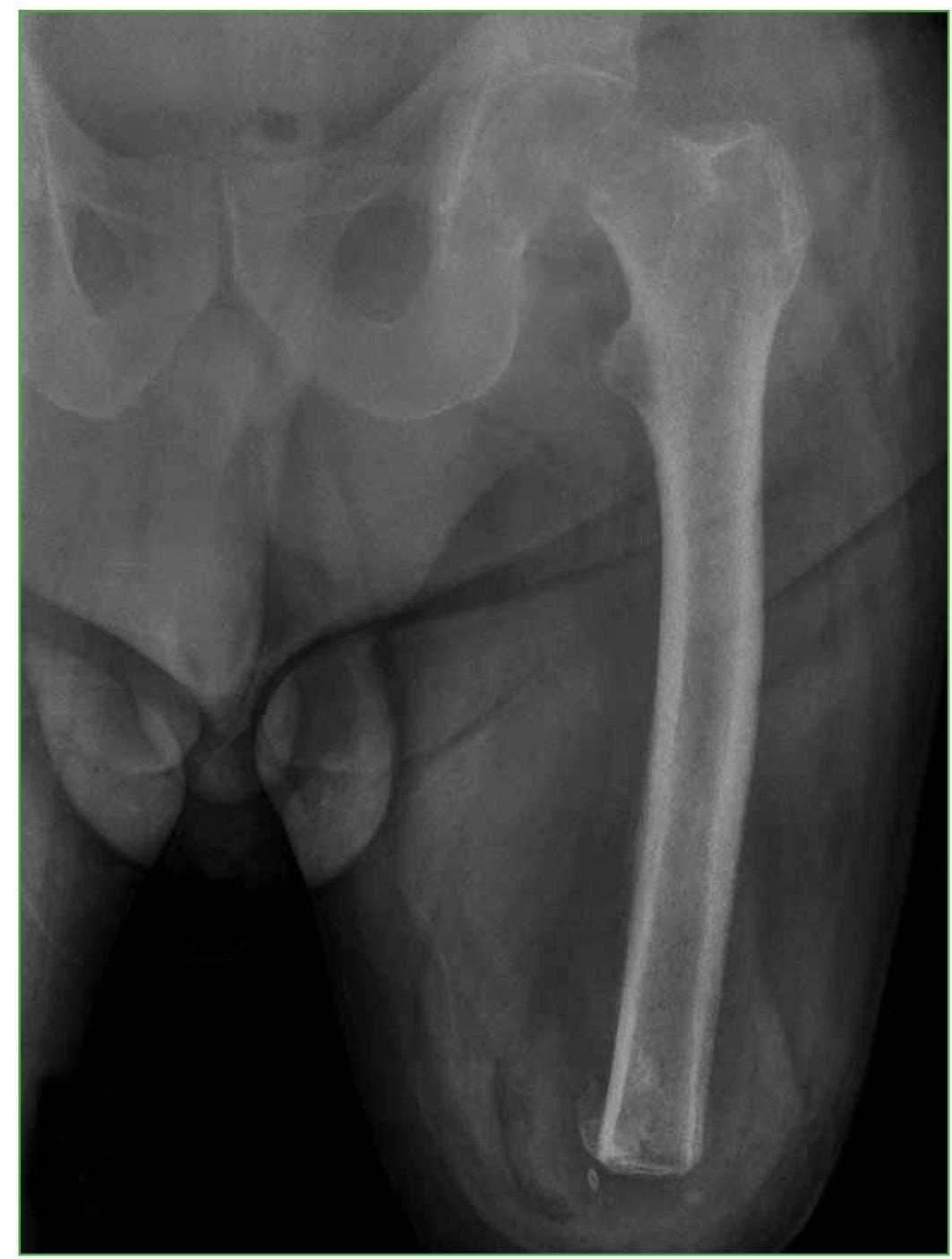

Figura 1. Radiografía anteroposterior de cadera izquierda, preoperatoria.

Se observa la fractura de cuello femoral sin desplazamiento. 
Bajo anestesia raquídea, el paciente fue colocado en decúbito lateral derecho. Se realizó una artroplastia total de cadera cementada mediante el abordaje de Gibson modificado y se colocó un clavo de Steinmann en el trocánter mayor para facilitar la dislocación/colocación y controlar la rotación del muñón. La artroplastia incluyó un implante femoral tipo Charnley con componente cefálico de $28 \mathrm{~mm}$ y cotilo Muller de $28 \mathrm{~mm}$ x $42 \mathrm{~mm}$ (Figura 2). En el posoperatorio, se administró profilaxis antibiótica por 48 h y terapia antitrombótica durante 15 días para prevenir complicaciones infecciosas y tromboembólicas.

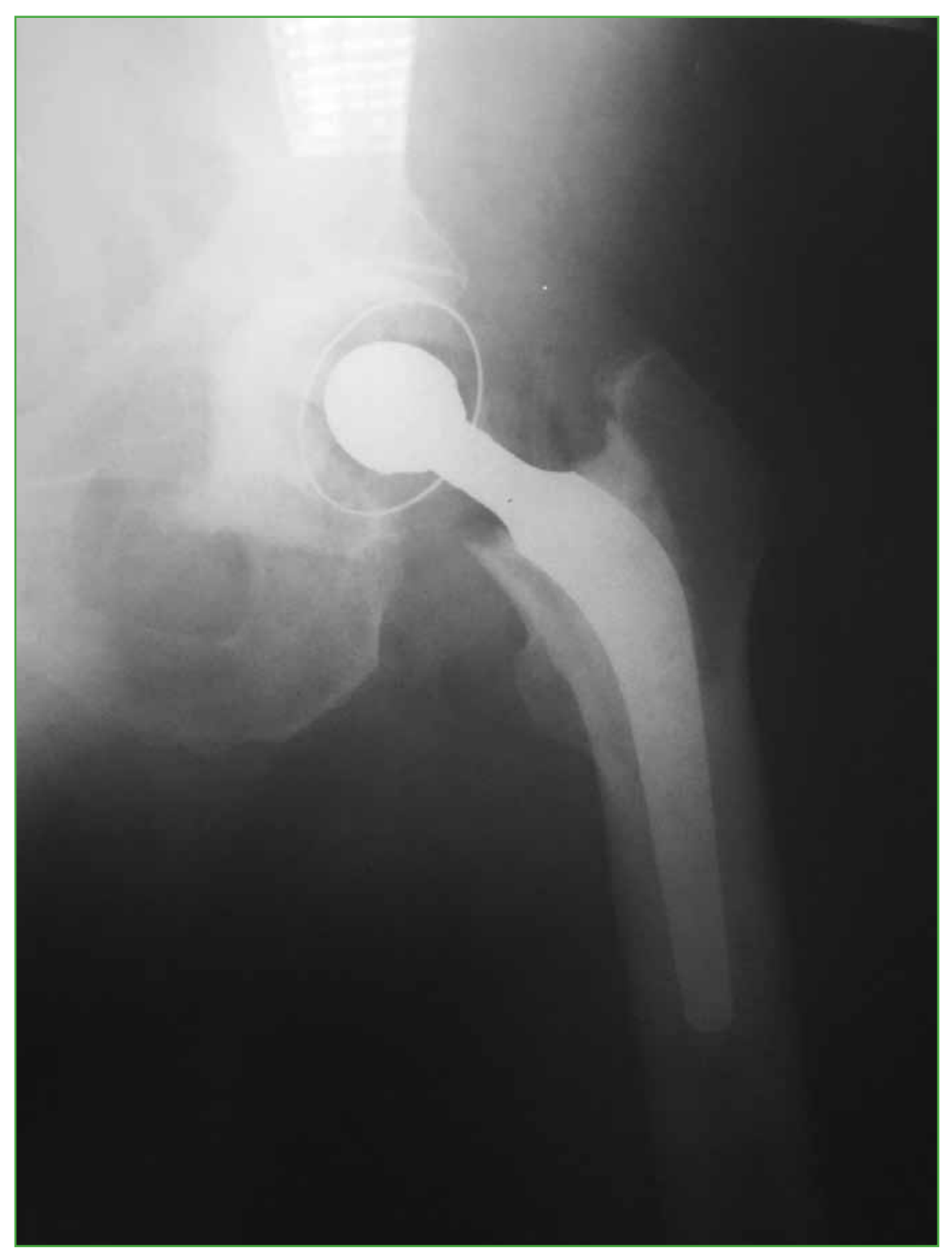

Figura 2. Radiografía anteroposterior posoperatoria satisfactoria. 
El paciente no tuvo complicaciones y se le permitió soportar su propio peso con el uso de la ortesis y la deambulación progresiva con muletas al tercer día de la cirugía.

Durante el seguimiento (15 meses), no hubo luxación de la prótesis ni complicaciones infecciosas. La herida quirúrgica estaba completamente cicatrizada y seca. El paciente tenía un rango de movilidad satisfactorio de la cadera: flexión $85^{\circ}$, rotación interna y externa $30^{\circ}$, abducción $40^{\circ}$ y aducción $20^{\circ}$. No sufría dolor, podía caminar con un bastón y realizar las actividades de la vida diaria de manera independiente, con el uso de su ortesis (Figura 3).
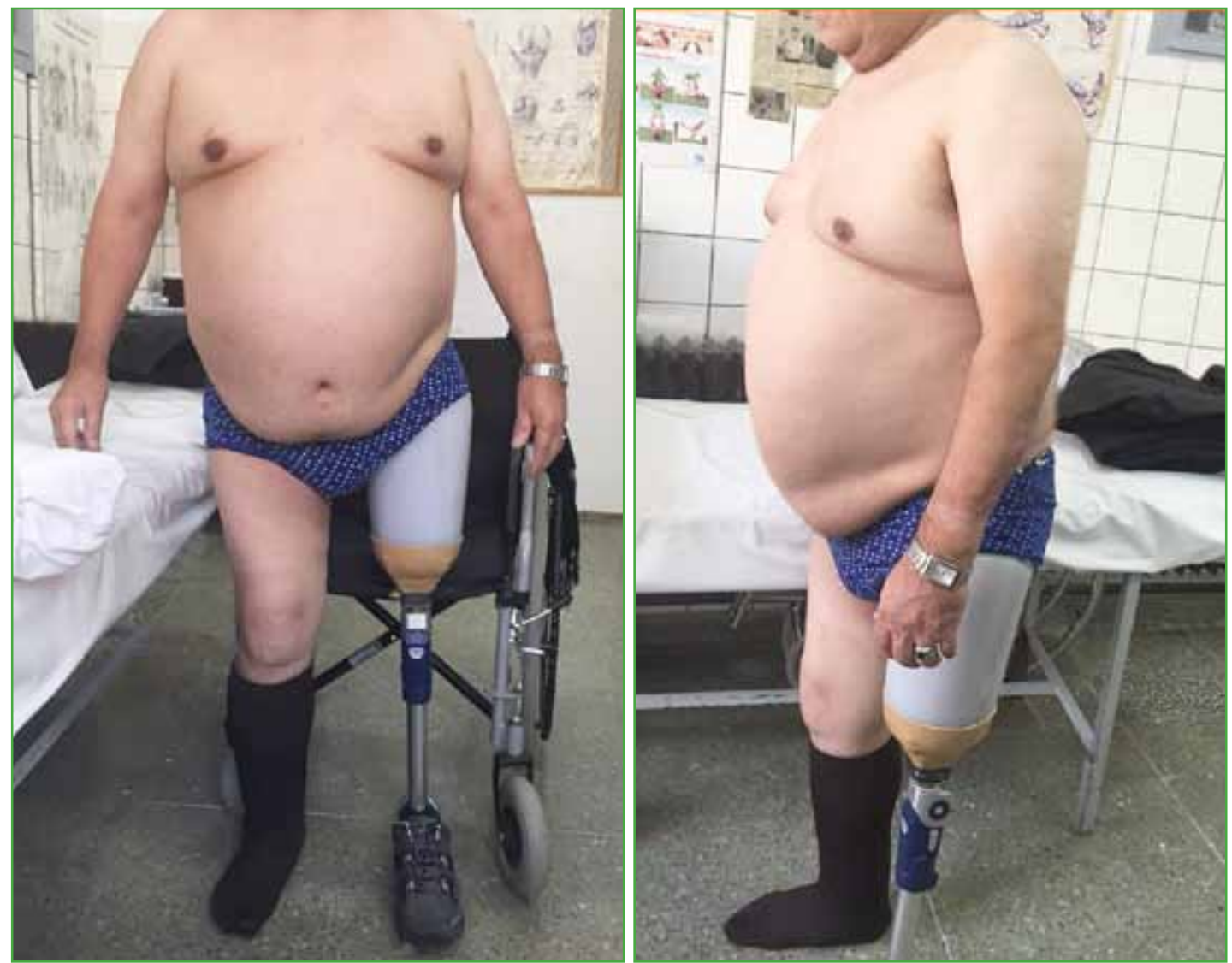

Figura 3. Aspecto clínico del paciente en el control a los 15 meses de la cirugía.

La radiografía anteroposterior de cadera no reveló ningún signo de migración de componentes ni aflojamiento de la prótesis. 


\section{DISCUSIÓN}

La artroplastia total de cadera es el procedimiento más utilizado en las fracturas de cuello femoral, principalmente en la población adulta. Este tipo de cuadro causa una alta tasa de morbilidad y mortalidad en el mundo; sin embargo, la artroplastia ha cambiado el pronóstico de la fractura medial de cadera. ${ }^{1,2}$

La fractura de cuello femoral en un miembro con amputación supracondílea no es una lesión común de encontrar y hay pocas publicaciones al respecto. ${ }^{1}$ Se estima que representa el $0,067 \%$ de todas las artroplastias de cadera. ${ }^{4}$

Se sabe que la amputación de un miembro provoca cambios osteoarticulares y musculares en el miembro afectado, así como también en el contralateral:2,3

- aumento de la osteoartritis tibiofemoral y femororrotuliana del lado no amputado.

- aumento de la osteoporosis del lado amputado.

- amiotrofia en el lado sano y el amputado, como resultado de las consecuencias anatómicas del acto quirúrgico y la pérdida de las inserciones musculares o su reimplantación en sitios no anatómicos.

Algunos estudios han demostrado que los pacientes con amputación de miembro inferior tienen un riesgo más elevado de sufrir una fractura de cadera homolateral por el alto índice de osteoporosis, así como por el brazo de palanca generado por la prótesis. ${ }^{1-4}$

Hay poca información publicada sobre el manejo de estas fracturas en pacientes con amputación supracondílea homolateral y, a pesar de que la artroplastia total de cadera es un método que brinda buenos resultados posoperatorios, la osteosíntesis con tornillos canulados de 7,5 mm es una buena opción terapéutica. ${ }^{6}$

La colocación apropiada del implante es esencial para obtener buenos resultados clínicos luego de una artroplastia de cadera. La exposición y la colocación del implante son procedimientos desafiantes en un miembro con amputación supracondílea. ${ }^{3,4}$ El uso de un clavo de Steinmann en el trocánter mayor es una buena técnica que permite controlar la rotación y generar la tracción necesaria del muñón para colocar el implante de forma correcta. ${ }^{7}$

Algunos autores ${ }^{1,2,4,6}$ describen otras técnicas para el control del muñón que pueden ser de gran ayuda, como el uso de fórceps óseo, clamp óseo, la colocación de tornillo de Schanz en dirección anteroposterior sobre el trocánter menor y la colocación de un clavo de Steinmann transfemoral.

Respecto al acto quirúrgico, la posición del paciente y el manejo del muñón fueron un gran desafío. Se consideró la edad del paciente, el tiempo de la cirugía, el abordaje que permitiera una buena exposición y el riesgo de fractura relacionado con la osteoporosis. ${ }^{1}$ Se eligió el abordaje de Gibson modificado, ya que permite una disección fácil y atraumática con reducción del tiempo quirúrgico y la pérdida hemática, proporciona una exposición excelente para la preparación y colocación de ambos componentes y permite la reconstrucción de los tejidos blandos, lo cual acelera la rehabilitación. ${ }^{8}$ Preferimos el uso de cemento en la artroplastia, porque tiene menos riesgo de aflojamiento de la prótesis en el hueso osteoporótico. ${ }^{1}$

\section{CONCLUSIONES}

El motivo de presentación de este caso es la baja incidencia de fracturas mediales de cadera en pacientes con amputación supracondílea homolateral y la poca información publicada sobre su manejo. El empleo de esta técnica quirúrgica, así como el uso del clavo de Steinmann para la manipulación del muñón redujeron el tiempo quirúrgico y se logró un buen resultado posoperatorio con el retorno a las actividades cotidianas normales del paciente.

Creemos que la artroplastia total de cadera es un excelente tratamiento para este tipo de fracturas, ya que permite una mejor rehabilitación con carga total de peso y deambulación temprana, a diferencia de la osteosíntesis, inclusive para fracturas no desplazadas.

Conflicto de intereses: Los autores no declaran conflictos de intereses. 


\section{BIBLIOGRAFÍA}

1. Boussakri H, Alassaf I, Hamoudi S, Elibrahimi A, Ntarataz P, ELMrini A, et al. Hip arthroplasty in a patient with transfemoral amputation: a new tip. Case Rep Orthop 2015; 2015: 593747. https://doi.org/10.1015/2015/593747

2. Masmoudi K, Rbai H, Fradj AB, Saâdena J, Boughattas A. Primary total hip replacement for a femoral neck fracture in a below-knee amputee. J Orthop Case Rep 2016;6(3):63-6. https://doi.org/10.13107/jocr.2250-0685.510

3. Pekmezci M, Nunley RM, Barrack RL. Technique for total hip arthroplasty in a patient with through-knee amputation. J Arthroplasty 2010;25(4):659.e1-3. https://doi.org/10.1016/j.arth.2009.03.011

4. Amanatullah DF, Trousdale RT, Sierra RH. Total hip arthroplasty after lower extremity amputation. Orthopedics 2015;38(5):e394-400. https://doi.org/10.3928/01477447-20150504-56

5. Nejat EJ, Meyer A, Sánchez PM, Schaefer SH, Westrich GH. Total hip arthroplasty and rehabilitation in ambulatory lower extremity amputees--a case series. Iowa Orthop J 2005;25:38-41. PMID: 16089069

6. Freitas A, Souto DRM, da Silva JF, Dantas BR, de Paula AP. Treatment of an acute fracture of the femoral neck in a young female adult with a transfemoral amputation: a case report. JBJS Case Connect 2015;5(3):e58. https://doi.org/10.2106/JBJS.CC.N.00119

7. Ma C, Lv Q, Yi C, Ma J, Zhu L. Ipsilateral total hip arthroplasty in patient with an above-knee amputee for femoral neck fracture: a case report. Int J Clin Exp Med 2015;8(2):2279-83. https://doi.org/1940-5901/IJCEM0004117

8. Varela-Egocheaga JR, Suárez-Suárez MA, Álvarez-Vega MA, Álvarez-Rico ME, Ferrero-Manzanal F, Montero-Díaz $\mathrm{M}$, et al. Abordaje posterior vs lateral en artroplastia total de cadera. Estudio prospectivo, aleatorio y ciego. Revista Española de Cirugía Osteoarticular 2005;40(223):126-33.

http://www.cirugia-osteoarticular.org/adaptingsystem/intercambio/revistas/articulos/161_126.pdf 